\title{
Modelo de incorporación de las pymes al comercio electrónico
}

\author{
An incorporation model of SMEs to e-commerce
}

\author{
Edwin Alberto Callejas \\ edwin.callejas@utec.edu.sv \\ Carlos Antonio Aguirre ${ }^{2}$ \\ Jorge Armando Aparicio ${ }^{3}$ \\ Investigadores Universidad Tecnológica de El Salvador
}

Recibido: 13/12/2014 - Aceptado: 02/07/2015

\begin{abstract}
Resumen
El documento que se presenta a continuación tiene como objetivo el desarrollo de un modelo que permita, de una forma sistematizada, incorporar las pymes al comercio electrónico a través del uso de la tecnología que actualmente poseen. Se presenta la base documental técnica de todos los elementos que integran el comercio electrónico, que deberán de ser considerados para la elaboración del modelo. Se realizó un estudio exploratorio de tipo descriptivo en el cual se pasaron encuestas a pymes que se dedican al área de servicios en el departamento de San Salvador, para lograr identificar la infraestructura tecnológica con la que cuentan actualmente y analizar la forma en que aquella puede facilitar la incorporación de la pymes al comercio electrónico a través del modelo propuesto.
\end{abstract}

\section{Palabras clave}

Pymes, comercio electrónico, modelo, infraestructura tecnológica.

\begin{abstract}
The objective of this document is the development of a model that allows the incorporation of SMEs to e-commerce in a sistematized manner by means of using the technology they currently possess.

The technical evidence base of all elements integrating e-commerce is presented; these shall be taken into account for the elaboration of the model.

A descriptive-type exploratory study was conducted. SMEs dedicated to offering services in the department of San Salvador were surveyed. Their purpose was to identify the technological infrastructure they currently have and to identify the way in which this can facilitate the incorporation of SMEs to e-commerce through the model proposed.
\end{abstract}

\section{Keywords}

SMEs, e-commerce, model, technological infrastructure.

Ingeniero de sistemas informáticos, Maestría en Computación, Maestría en Docencia Universitaria, actualmente docente investigador de la Escuela de Informática. Correo electrónico edwin.callejas@utec.edu.sv

2 Licenciado en Administración con especialidad en Computación, Maestría en Computación, actualmente docente investigador de la Escuela de Informática. Correo electrónico carlos.aguirre@utec.edu.sv

Ingeniero en Electrónica, Director de la Escuela de Informática. Correo electrónico jorge.aparicio@utec.edu.sv 


\section{Introducción}

Esta investigación tiene como propósito el desarrollo de una propuesta de modelo que les permita, a las pymes, seguir un patrón para facilitarles su incorporación al comercio electrónico, utilizando la infraestructura tecnológica que poseen o que deben de adquirir para lograr ese propósito.

El comercio electrónico, actualmente, es una realidad como medio que permite a las empresas llegar a ofrecer sus productos y servicios a una mayor cantidad de clientes, aumentando sus ingresos al incrementar sus transacciones. El conocimiento de las condiciones actuales en el país que logre garantizarles, a las pymes, el medio electrónico para realizar sus operaciones hará que disminuya la incertidumbre actual sobre esta forma de hacer negocios. Entre las condiciones que se analizarán se encuentran la tecnología necesaria, la seguridad, el recurso humano y los clientes (Kaba, 2008).

\section{La tecnología necesaria}

Es importante que las pymes que deseen incorporarse al comercio electrónico puedan realizar un autoestudio sobre sus recursos tecnológicos actuales, los que necesitan obtener y los que tienen que desarrollar, para cumplir con las expectativas que demanda esta iniciativa. Entre estos recursos se pueden mencionar sistemas transaccionales que apoyen cada una de las operaciones del negocio, tales como sistemas de facturación, de clientes, de marketing, de inventario, entre otros. Además, será necesario revisar las infraestructuras tecnológicas de servidores y de comunicación de datos que darán soporte a cada uno de los procesos del negocio y que permitan recolectar toda la información relacionada con las operaciones del negocio, que posteriormente se analizaría como entrada de información al modelo.

\section{La seguridad}

La primera seguridad debe de ser la jurídica y la de la información procesada por los sistemas, esto es, en primera instancia, que debe existir un marco regulatorio que garantice las realización de estas operaciones a través del medio electrónico; y la segunda se refiere a la implantación de mecanismos que le garanticen, a las pymes, la confiabilidad de los sistemas para transferir y compartir información crítica del resultado del procesamiento de operaciones por medios electrónicos.

\section{El recurso humano}

Es necesario conocer las competencias tecnológicas y del negocio del recurso humano de las pymes para implantar y operar los procesos y procedimientos que demandará la implantación del modelo. Se necesitara información de perfiles técnicos o necesidades de capacitación del personal de las pymes en sus diferentes áreas que formarán parte del modelo.

\section{Los clientes}

Son actores directos del modelo, ya que serán sus datos los que serán recolectados a través de medios tecnológicos y analizados para extraer información significativa de su estudio, lo cual podría asociarse a invasión de privacidad por parte de la empresa para ofrecer alternativas de hacer negocios, es decir, este factor puede llegar a ser determinante para lograr que la solución sea funcional o no, en el sentido de determinar quién es el dueño de los datos en internet.

\section{Justificación del estudio}

Muchas empresas actualmente poseen infraestructura tecnológica, procesos sistematizados, estrategias de marketing, pero no son efectivas en capitalizar las bondades que ofrece el comercio electrónico, ya que no logran proporcionarle al potencial cliente información oportuna y significativa sobre sus necesidades en el momento deseado, ya sea por una toma de decisiones tardía o por no disponer de la información necesaria para ofrecerle cuando se requiere. Se pretende que las pymes hagan uso de tecnología para diseñar estrategias que les permitan incorporarse al comercio electrónico, utilizando la información que se genera en la web.

En la actualidad, la tecnología de macrodatos, o de inteligencia de datos, está tomando cada vez más realce dentro del mundo de los negocios y las estrategias. El 
conocimiento de esta tecnología puede ser aprovechada por cualquier empresa, con el fin de tener una mejor forma de brindar sus productos y servicios. El aprovechamiento de esta tecnología le permitirá a las pymes conocer más de cerca a sus clientes, prestarles un mejor servicio, mejorar la calidad de sus productos, generar oportunidades para ingresar a nuevos mercados, completar su portafolio de clientes, entre otras tareas que generen beneficios al negocio. Por lo tanto, la investigación sobre la tecnología de macrodatos dentro de las pymes está basada en los siguientes indicadores: conocer la infraestructura tecnológica que tienen actualmente; la utilización y las herramientas que son parte esencial en el intercambio comercial de la misma; y conocer los puntos en los cuales se puede mejorar ampliar o remplazar aquellos que así lo requieran para agilizar los procesos de sus negocios.

\section{Objetivos}

a) Investigar la tecnología de macrodatos en el contexto del comercio electrónico y las pymes.

b) Identificar los diferentes actores participantes en el comercio electrónico, de tal forma que permita construir un modelo que incorpore a las pymes al comercio electrónico.

c) Determinar el escenario actual en el área de comercio de las pymes.

d) Elaborar un modelo de incorporación de las pymes al comercio electrónico.

\section{Las pymes, el comercio electrónico y la tecnología de macrodatos}

\section{Las pymes y el comercio electrónico}

La pequeña y mediana empresa, que normalmente se conoce como pymes, es un grupo comercial con características distintivas y tiene dimensiones con ciertos límites ocupacionales y financieros prefijados por los Estados. Las pymes se refieren a la pequeña y mediana empresa de El Salvador y son agentes con lógicas, culturas, intereses y espíritu emprendedor específicos NIIF(Niif El Salvador, 2014).

Las pymes, a escala nacional, son uno de los actores claves del crecimiento salvadoreño, siendo reconocidas también como fuentes directas de empleo, en el producto interno bruto (PIB) y en el comercio exterior. La innovación tecnológica nacional e internacional en que se mueven los negocios plantea importantes desafíos a las pymes. Se dividen en dos grandes segmentos: las sociedades y las empresas de hogares (Martínez, 2013).

En la tabla 1 se presenta la clasificación de las pequeñas y medianas empresas en El Salvador.

Tabla 1.

Clasificación de las pymes.

Cámara de Comercio e Industria de El Salvador

(Minec, 2012)

\begin{tabular}{|l|c|}
\hline Clasificación & Porcentaje de establecimiento \\
\hline Pequeña & 7,54 \\
\hline Mediana & 1,50 \\
\hline Total pymes & 9,04 \\
\hline
\end{tabular}

Las pymes son las empresas que mantienen un total de ventas anuales que oscilan entre los 457.1428 .50 hasta los 6.8571 .28 dólares y un total de entre 11 y 199 empleados. El Salvador tiene más de medio millón de pymes. Se calcula que emplean al $66 \%$ de la población económicamente activa y aportan el $44 \%$ del PIB (Minec, 2012).

Existe un total de 17.5178 empresas en El Salvador clasificadas como pymes, de las cuales se hace una distribución generalizada por cada uno de los sectores, cuyo porcentaje está representado en la tabla 2. 
Tabla 2.

Distribución por sector de las Pymes. Cámara Salvadoreña de Comercio e Industria de El Salvador

(Minec, 2012).

\begin{tabular}{|l|c|}
\hline \multicolumn{1}{|c|}{ Sector } & Porcentaje \\
\hline Industria & 13 \\
\hline Comercio & 66 \\
\hline Servicios & 18,4 \\
\hline Transporte & 2,3 \\
\hline Construcción & 0,3 \\
\hline Electricidad & 0,05 \\
\hline Agroindustria & 0,04 \\
\hline Total & $\mathbf{1 0 0}$ \\
\hline
\end{tabular}

De los anteriores sectores, que establece la tabla 2, los que interesa estudiar son los de comercio y de servicios.

\section{Ventajas del comercio electrónico}

La web ofrece a los proveedores la oportunidad de relacionarse con un mercado totalmente interactivo, donde las transacciones, transferencias, inventarios y recolección de datos, entre otras actividades, pueden realizarse en línea. Esto permite que las empresas puedan incrementar su eficiencia, disminuyendo el tiempo de estas operaciones; automatizar los procesos de administración; acelerar la entrega de productos y mejorar la distribución (Gerencia, 2014).

Entre otras ventajas, el comercio electrónico también permite que los consumidores cuenten con una plataforma de compra durante las 24 horas del día y que las empresas se introduzcan en un mercado focalizado, a la medida de las necesidades de los clientes, al tiempo que disminuyen sus costos. Esta modalidad les brinda la oportunidad, a las organizaciones, de llegar a aquellos mercados geográficamente inalcanzables de una manera rápida y eficaz, y de entrar en un nuevo segmento de consumidores (Gerencia, 2014).

\section{Tecnologías de macrodatos}

Macrodatos es un término aplicado a conjuntos de datos que superan la capacidad del software habitual, para ser capturados, gestionados y procesados en un tiempo razonable. Se considera un conjunto de datos que crecen rápidamente y que no pueden ser manipulados por las herramientas de gestión de bases de datos tradicionales. (Aguilar, 2013). Cada tipo de dato contenido dentro de los macrodatos es información valiosa, sin embargo, depende de la finalidad que se busque alcanzar, ya que no toda la información en ese ámbito es idónea para lograrlo; es por esta razón que deben elegir muy bien. Los tipos de datos están clasificados, como se muestran en la figura 1, y provienen de muchas fuentes, siendo las principales las que mencionan abajo.

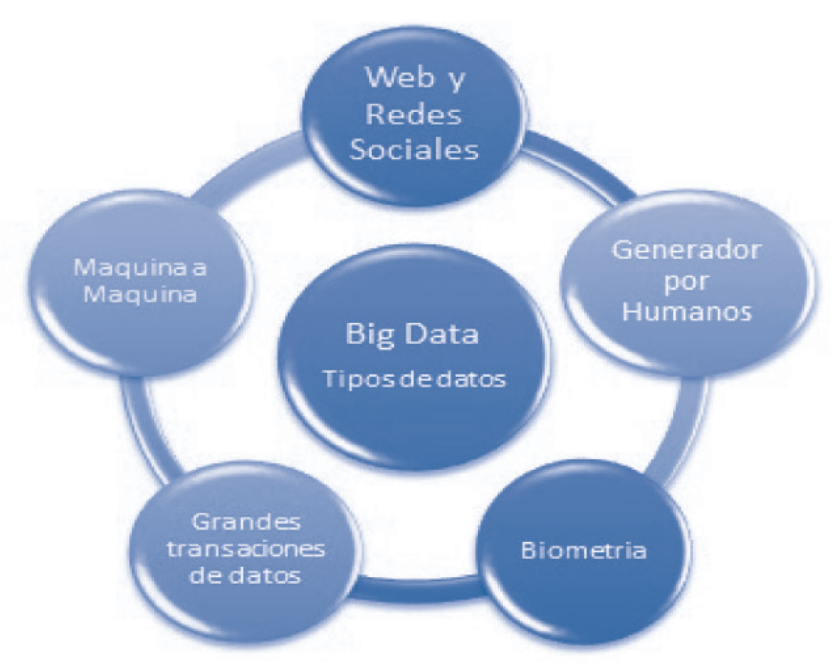

Figura 1.

Tipos de macrodatos

Fuente: Sitio web de IBM (IBM, 2012)

a) Web y redes sociales. Se refiere a información contenida en la web que se divulga a través de las redes sociales, tales como Facebook, Twitter, Linkedın, blogs, entre otros sitios donde hay intercambio de media (fotos, música, vídeos, documentos, ubicación) (IBM, 2012).

b) Máquina a máquina (machine-to-machine, M2M). Se refiere a las tecnologías que permiten la conexión entre dispositivos. utiliza dispositivos como sensores o medidores que capturan algún evento en particular (velocidad, temperatura, presión, variables meteorológicas, variables químicas como la salinidad), los cuales transmiten a través de redes alámbricas, inalámbricas o híbridas a otras aplicaciones que traducen estos eventos en información significativa (IBM, 2012). 
c) Grandes transacciones de datos. Se refiere a registros de facturación, en telecomunicaciones, registros detallados de las llamadas (CDR), entre otros, realizados por grandes empresas. Estos datos transaccionales están disponibles en formatos tanto semiestructurados como no estructurados (IBM, 2012).

d) Biometría (biometrics). Es aquella que se obtiene de hueIlas digitales, escaneo de la retina, reconocimiento facial, genética como el ADN (ácido desoxirribonucleico). Los datos biométricos han sido información importante para las agencias de investigación (IBM, 2012).

e) Generado por humano (human generated). Información que generan a diario las personas son cantidades de datos, como los que guarda un call center al establecer una llamada telefónica, notas de voz, correos y documentos electrónicos, estudios médicos digitalizados, historiales de navegación y preferencias en la web (IBM, 2012).

Factores que inciden en la implantación de un modelo de incorporación de las pymes al comercio electrónico

\section{Recursos tecnológicos}

Se refiere a los recursos tecnológicos que serán necesarios para poder implantar la tecnología, esto es, en los ámbitos de software y hardware, por ejemplo, sistemas de información transaccionales, software especializado de análisis, hardware especializado para la captura y el análisis de información.

\section{Recursos humanos}

Se debe analizar este factor desde tres perfiles: el cliente, que proporcionará información y que confía en que no será vulnerada su privacidad; el usuario, si está preparado para explotar al máximo la información que pueda obtener del análisis de datos; y el personal de IT, que se encargara de implantar las estrategias de búsqueda de información y generar valor agregado a la empresa a través del análisis de las operaciones y de sus clientes.

\section{Seguridad de la información}

El concepto de seguridad de la información no debe ser confundido con el de seguridad informática, ya que este último solo se encarga de la seguridad en el medio informático, pero la información puede encontrarse en otros medios o formas y no solo en medios informáticos (Castro, 2014). La información es poder; y según las posibilidades estratégicas que ofrece tener acceso a cierta información, esta se clasifica como se presenta a continuación:

i. Es indispensable para la operación de la empresa.

ii. Es un activo de la empresa; y muy valioso.

iii. Debe de ser conocida por las personas autorizadas.

En la seguridad de la información hay que considerar lo siguiente:

- La materialización de vulnerabilidades identificadas, asociadas con su probabilidad de ocurrencia, amenazas expuestas, así como el impacto negativo que ocasione a las operaciones de negocio (WCruzy, 2014).

- La forma de protección contra los riesgos (WCruzy, 2014).

\section{Metodología}

La ausencia o desconocimiento de una metodología que permita a las pymes incorporarse al comercio electrónico es un factor que incide negativamente para que asuman ese aparente riesgo de una forma ordenada y sistematizada, más bien lo hacen basadas en experiencias compartidas con sus pares o a través de asumir el riesgo a innovar en el proceso aun cuando, los beneficios pueden ser muchos, aun así lo consideran aventurero.

\section{Método}

En el contexto teórico, con la investigación exploratoria, se realizó una recopilación de datos a través de encuestas y entrevistas a usuarios y desarrolladores buscando inducir con ideas sobre los factores que están inmersos en la implantación de un modelo de incorporación de las pymes 
al comercio electrónico, como, además, sensibilizando a los participantes sobre los beneficios que esta tecnología le puede ofrecer a las pymes en la generación de mayor participación de sus operaciones a través del comercio electrónico. Dado que se carece de información suficiente y de conocimientos previos sobre el objeto de estudio, resulta lógico que la formulación inicial del problema sea imprecisa. En este caso la exploración permitió obtener datos y elementos que ayudaron a conducir a formular con mayor precisión la creación de los instrumentos de investigación y de procesamiento de datos, y su respectivo análisis.

\section{Participantes}

Se seleccionaron las empresas que se clasificaron como pymes de los sectores comercio y servicios del municipio de San Salvador, así como a un conjunto de expertos que a partir de su conocimiento y experiencia podrían apoyar con información valiosa el desarrollo de la investigación.

\section{Instrumentos de recolección de información}

Para la elaboración de este trabajo fue necesario utilizar algunos recursos tecnológicos y materiales integrados en las pruebas, como el programa Microsoft Office Excel 2013, encuestas, entrevistas y otros recursos. Los miembros del grupo diseñaron los instrumentos; se pidió que reflexionaran y escribieran cuatro factores que constituyen el comercio electrónico y la tecnología de los macrodatos, y los factores que inciden en la implantación de los diferentes elementos del modelo.

Cada participante anotó los factores, los cuales en su conjunto se valoraron y clasificaron para listar un serie de preguntas agrupadas en categorías, aunque con la conciencia de que, en alguna medida, unas estaban implícitas en otras, por lo que se debió depurar la lista, teniendo como resultado el diseño de encuestas y entrevistas, además, dichos factores se seleccionaron en base a criterios a los expertos que apoyaran la fase de validación de información.

\section{Procesamiento de datos}

Los datos se procesaron calculando la frecuencia de las respuestas a las diferentes preguntas planteadas y luego se sacaron valores porcentuales que permitieron mostrar, en términos relativos, valores de comparación respecto al total de la muestra que respondieron la pregunta; esto permitirá ver la tendencia de aceptación de cada opción de respuesta.

\section{Presentación de resultados}

Con las encuestas realizadas, se busca medir el grado de utilización y aprovechamiento de la tecnología de macrodatos para realizar comercio electrónico por parte de las pymes. A continuación se presentan los resultados obtenidos de las encuestas realizadas durante los meses de octubre-noviembre del 2013. El análisis de las frecuencias y su distribución porcentual de participantes en las respectivas preguntas quedan reflejados en las siguientes figuras.

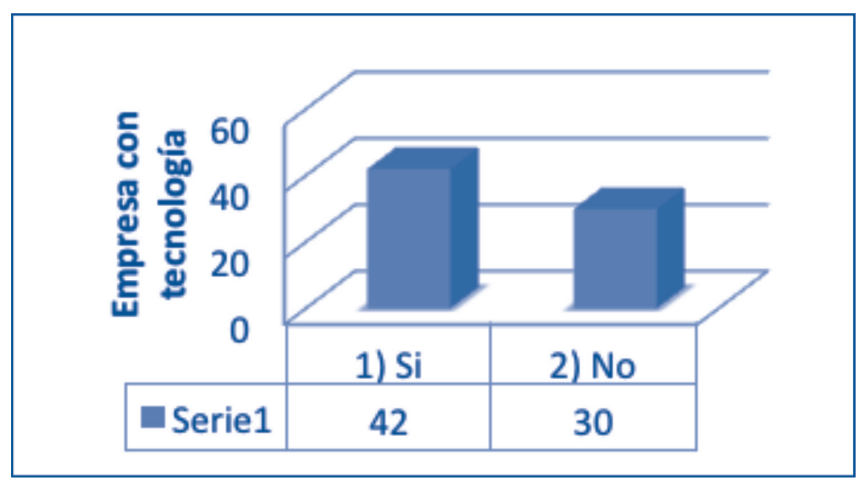

Figura 2.

¿Cuenta su empresa con tecnología para el análisis de la información?

Como observamos en la figura 2, la diferencia entre las empresas que poseen tecnología para análisis de la información es relativamente baja en relación con las que no la poseen. Claramente se muestra que la cantidad de empresas que tienen esta tecnología es mayor. Sin embargo, aunque la cantidad de empresas que no disponen de este tipo de herramientas es parcialmente baja; la diferencia puede ser un indicador de desconocimiento o de poco interés en la adquisición de herramientas para el análisis de la información.

Con respecto a los medios digitales de comunicación de las empresas encuestadas, se muestra la figura 3 en la 
que se refleja que las empresas que cuentan con las redes sociales están con un $35 \%$. El segundo mayor porcentaje de la gráfica es resultado de las empresas que poseen su canal en Comunicación interactiva, seguido por las empresas que poseen medios electrónico.

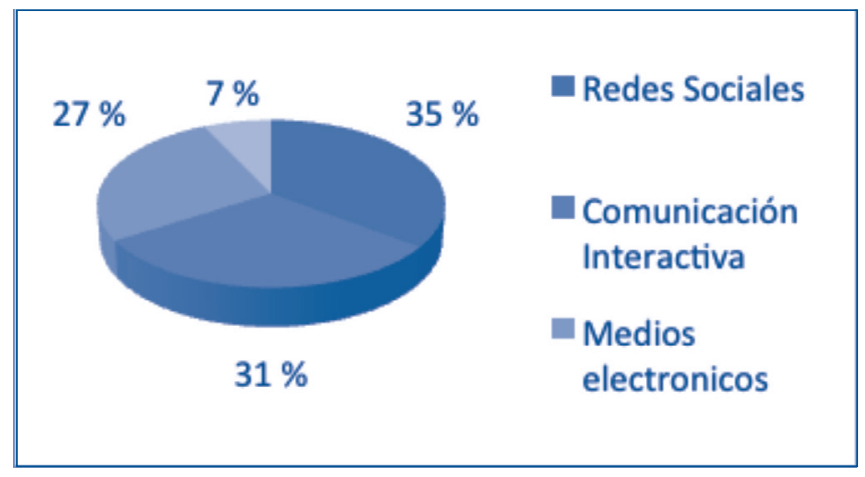

Figura 3.

¿Cuáles medios digitales de comunicación se utilizan en su empresa?

La figura 4 muestra los resultados obtenidos de la frecuencia con la que las empresas encuestadas usan los medios de comercio electrónico, el cual indica que aproximadamente el 77 \% utilizan los medios electrónicos como herramientas para comerciar, principalmente las redes sociales, para dar a conocer sus productos y servicios. Estos porcentajes aumentarían a $89 \%$ si se consideran aquellas que también lo utilizan de forma ocasional. En ese sentido, se muestra cada vez un mayor interés por su utilización.

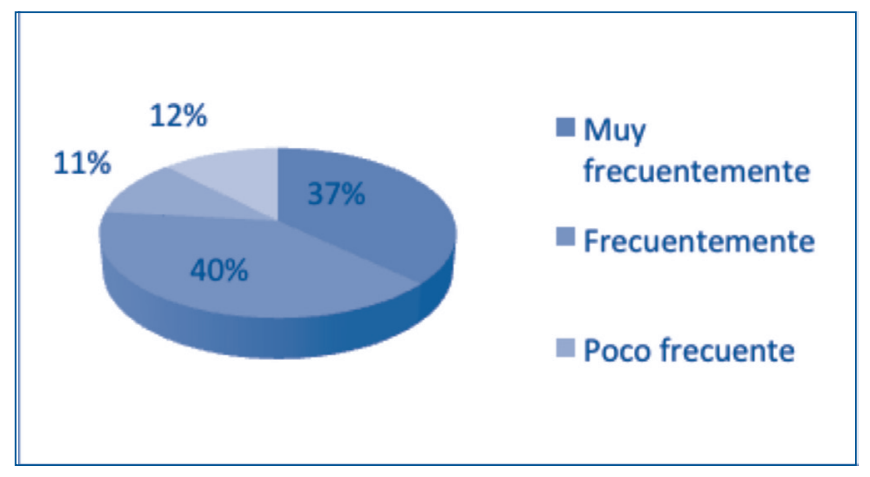

Figura 4.

¿Con qué frecuencia su empresa utiliza internet, páginas webs, blogs, correo electrónico y redes sociales (Facebook, Twitter y otros) como herramienta para promocionar?
En la figura 5 se ve el grado de aceptación en el uso de las redes sociales y otros para el crecimiento en las empresas encuestadas, con un porcentaje de $71 \%$. El porcentaje de quienes a su criterio no consideran que los medios electrónicos de comunicación sean de ayuda para el crecimiento de su empresa fue de $9 \%$; seguido de los que dudan, que fue de $7 \%$.

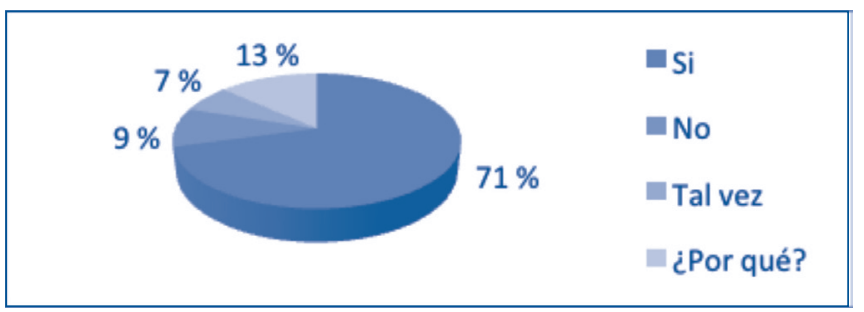

Figura 5.

De acuerdo con su criterio, ¿considera usted que las redes sociales, internet, Facebook, Twitter, página web, blogs, correo electrónico, juegan un papel importante para el crecimiento de clientes en la empresa?

En la figura 6 se observa cuáles son los criterios utilizados para otorgar descuentos en los productos y promocionar dentro de su empresa.

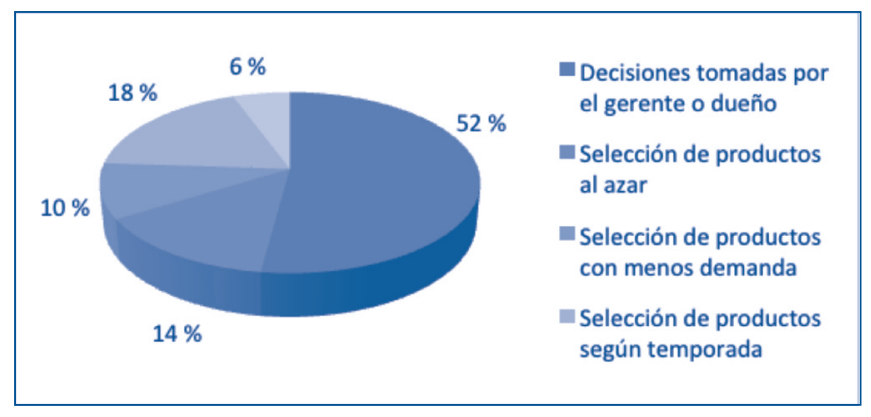

Figura 6.

Actualmente, ¿en qué se basan para la creación de las promociones y descuentos en los productos?

La figura 7 recoge los resultados alcanzados para conocer las herramientas o técnicas utilizadas por las empresas encuestadas para informar acerca de productos nuevos o promociones a sus clientes. En la actualidad las redes sociales son comúnmente utilizadas para promocionar y 
ofrecer productos o servicios a escala mundial, logrando el primer lugar con el $52 \%$; seguido de los medios electrónicos, con el $21 \%$.

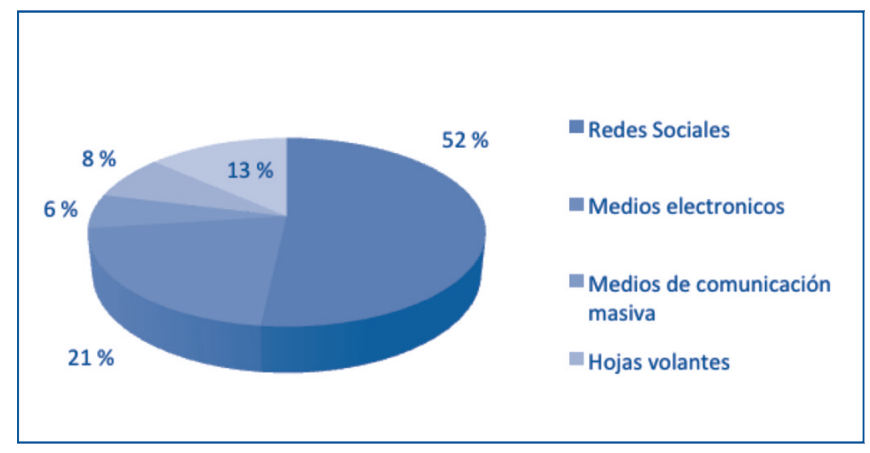

Figura 7.

¿Qué herramientas, técnicas o métodos utiliza la empresa para dar a conocer las promociones y los nuevos productos a sus clientes existentes y potenciales?

De acuerdo con los resultados del estudio con respecto a si el análisis de datos, información de clientes, productos, promociones y datos importantes lograrían ayudar a mejores resultados financieros a la empresa, se observa en la figura 8).

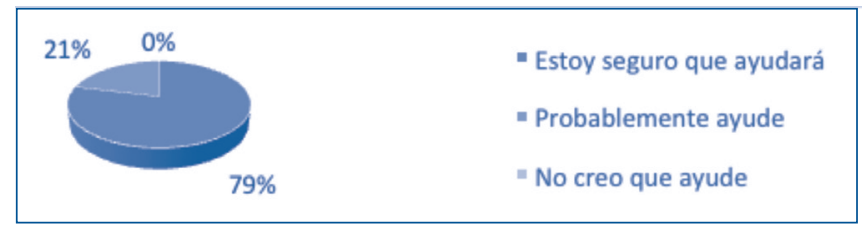

Figura 8.

¿Considera que el análisis de datos, la información de clientes, productos, promociones y datos importantes pudieran ayudar a su empresa a conseguir los resultados esperados?

En su mayoría, las empresas tomadas como muestra para este estudio consideran que es de gran beneficio realizar análisis de datos para el desarrollo y crecimiento de su entidad, según se muestra en la figura 9.

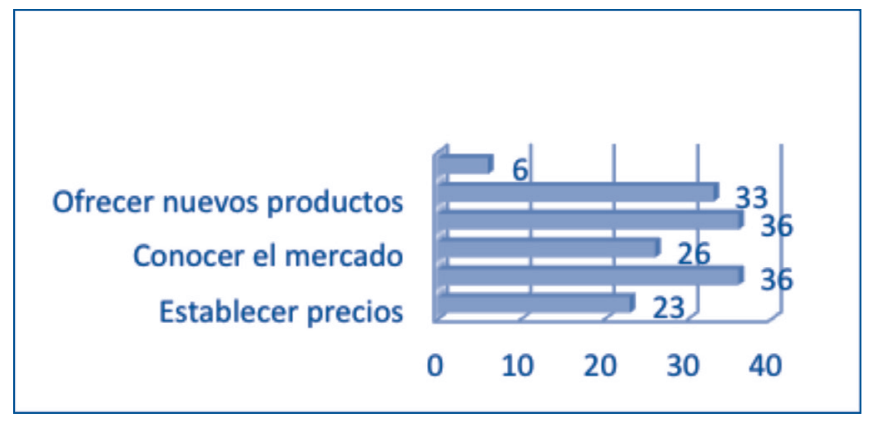

Figura 9.

Si su empresa realizara análisis de datos ¿Cuáles considera usted que serían los principales factores que se verían beneficiados en su empresa?

A continuación, una clara representación, en la figura 10, de la consideración de ventaja competitiva con referencia al análisis de datos dentro de las empresas encuestadas; consideran una ventaja competitiva el $49 \%$; seguido de una relativamente buena, con $34 \%$, los cuales son los rangos más altos. Luego, un $10 \%$ considera que es muy poca ventaja; seguido de los que no lo consideran una ventaja, con el $7 \%$.

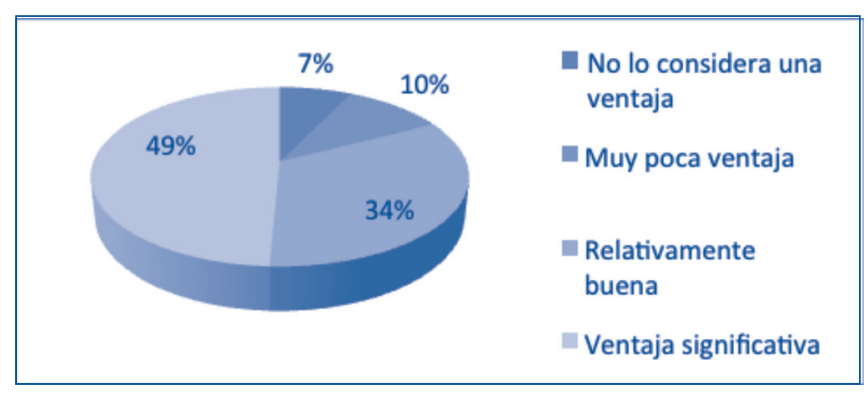

Figura 10.

¿En qué medida considera que el análisis de datos resultaría en una ventaja competitiva para su empresa?

La figura 11 muestra los resultados obtenidos, que serían los factores que impedirían la implantación de un modelo de análisis de datos. El mayor porcentaje contemplado, $37 \%$, considera que la falta de recursos financieros no les permitiría instaurar una herramienta de análisis; seguido del $20 \%$, que es el que estima que la falta de experiencia en el tema sería un impedimento. 
En los siguientes rangos de puntos porcentuales se observa que la muestra tomada tiene registrados con $18 \%$ a los que no saben sobre el tema o no responden; seguido de $14 \%$, que consideran que no cuentan con la infraestructura necesaria; y para finalizar, con $11 \%$ a los que consideran que el análisis de datos e información no es aplicable al giro de su empresa.

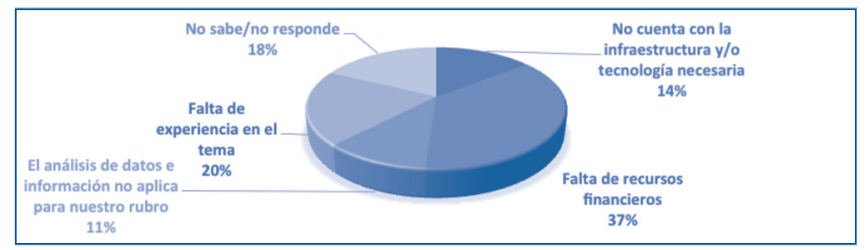

Figura 11.

¿Qué factores impedirían la implantación de un modelo de análisis de datos e información en su empresa?

El estudio muestra el número de empresas que estarían interesadas en adquirir el software y la herramienta de análisis, según la figura 12.

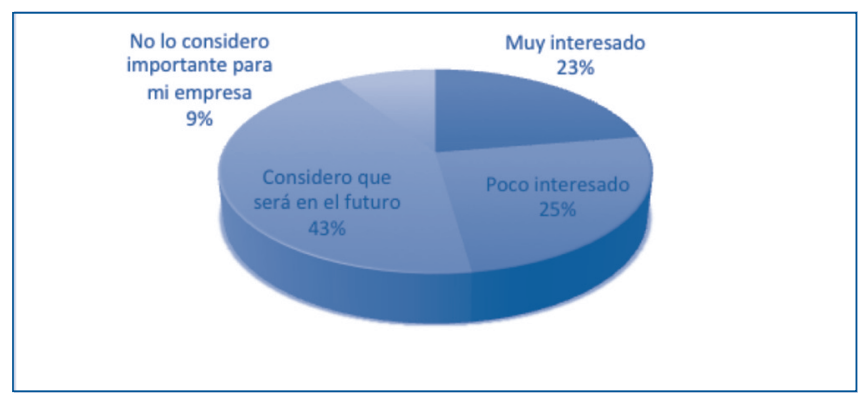

Figura 12.

¿Estaría interesado en adquirir software que le permita a su empresa realizar análisis de datos?

Los resultados obtenidos se representan en la figura 13, indicando los aspectos que se considera se deben tomar en cuenta para la adquisición de una herramienta para el análisis de datos de las empresas encuestadas. Es muy importante que las empresas valoren la asesoría por parte de profesionales, el soporte 7/24 y que sea una marca reconocida. A partir de lo anterior, se podrían interpretar dichos resultados debido a la desconfianza que tienen las empresas hacia esta tendencia tecnológica porque deben entregar los aspectos de marketing, ya que consideran que es muy alto el riesgo de compartir sus datos confidenciales. Quizá consideren que deben de apoyarse de experiencias de éxitos de otras empresas; y de que sea una marca reconocida y de éxito en el medio.

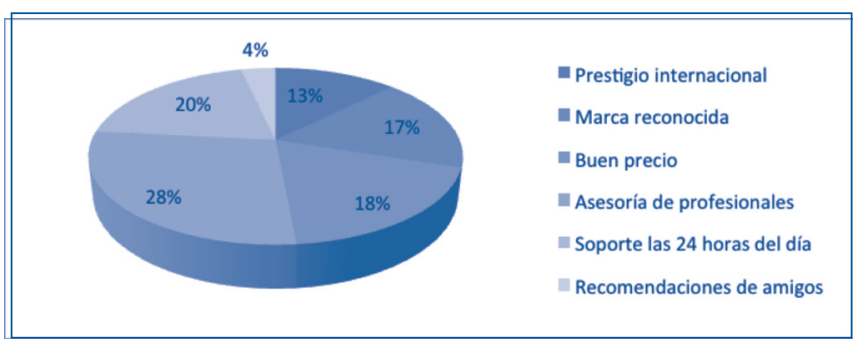

Figura 13.

¿Qué aspectos considera que se deben tomar en cuenta para la adquisición de una herramienta para el análisis de datos dentro de su empresa?

La figura 14 muestra los resultados obtenidos en referencia a la cantidad, en términos financieros, que estarían dispuestos a pagar para adquirir un software de análisis de datos.

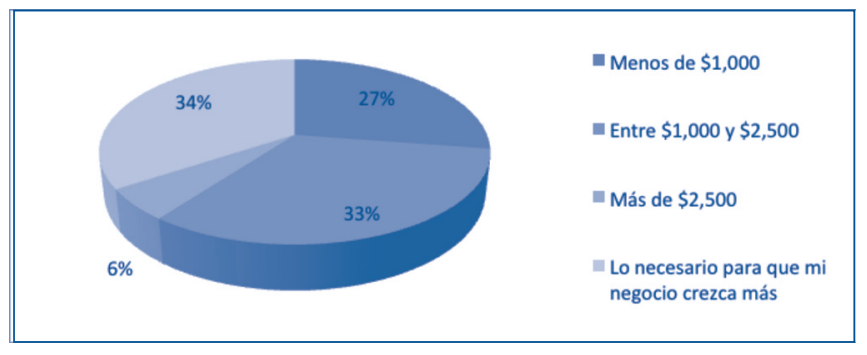

Figura 14.

Considerando que el análisis de datos mejorara los ingresos de su negocio, ¿cuánto sería la inversión que estaría dispuesto a pagar para adquirir un software de análisis de datos?

En la figura 15 se observa cómo prevalece la mayoría, según la muestra entrevistada, en el apartado Solicitar firmar contrato de confidencialidad, con un rango de $62 \%$; seguido de $51 \%$, que consideran que se deben establecer políticas de seguridad; y de los que sostienen que el desarrollo de la herramienta es oportuno y que sea dentro de la empresa, 
$9 \%$; y $8 \%$, los que solicitarían recomendaciones de otras empresas.

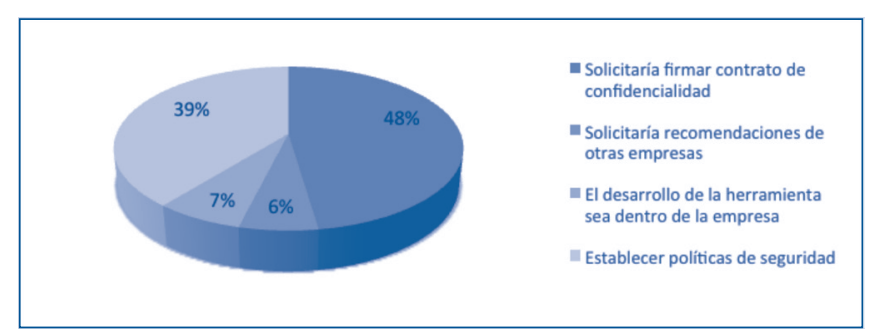

Figura 15.

¿Qué medidas de seguridad exigiría para proteger la información de su negocio al momento de contratar a una empresa que se encargue de la creación de software de análisis de datos?

\section{El modelo de incorporación al comercio electrónico}

El modelo expone los actores y elementos que se deben considerar para propiciar una ruta de incorporación de las pymes al comercio electrónico, a través del uso de los macrodatos, como se ilustra en la figura 16.

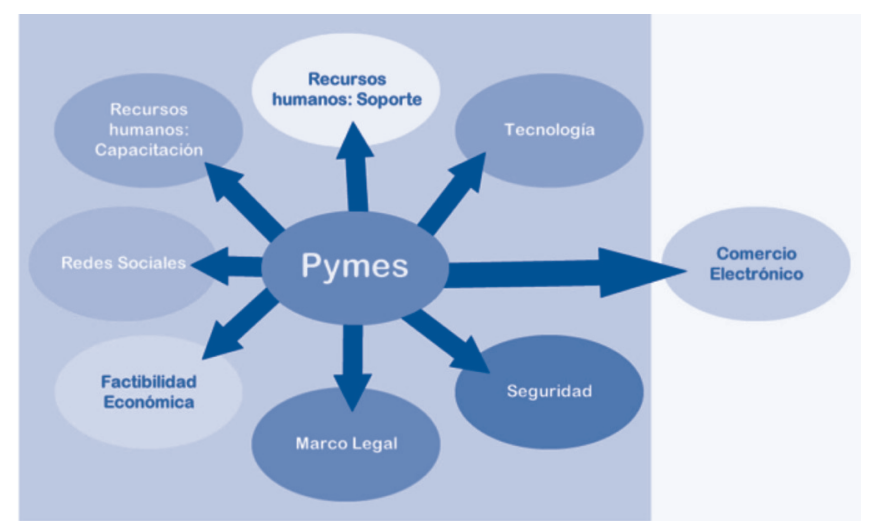

Figura 16.

Modelo de incorporación de las pymes al comercio electrónico. Fuente: Elaboración propia.

\section{Elementos que se deben considerar en el modelo}

a) Capacitación de personal. Es importante contar con personal capacitado en todas las áreas integradas para operar el modelo, específicamente en las áreas de tecnología, mercadeo y operaciones en la empresa, sobre el cumplimiento de los objetivos propuestos. b) Sistemas de información transaccionales. Es vital que la pymes cuenten con sistemas mecanizados en los procesos, tales como facturación, inventario, promociones y gestión de clientes.

c) Medios seguros de transferencia de datos. Se refiere a que la empresa debe de implantar mecanismos de seguridad en sus transacciones para establecer confiabilidad en el procesamiento de la información; esto pasa por adoptar leyes, reglamentos, instructivos y procedimientos establecidos para la realización del análisis de la información

d) Capacidades de recursos informáticos. Una inversión necesaria pasará por actualización: adquisición de equipos; por actualización: adquisición de software, por lo que la empresa debe estar en la disponibilidad de dicha inversión

\section{Recursos humanos}

\section{Perfil del recurso humano}

a) Administrador de la base de datos (DBA). Contando con las competencias de conocer el negocio, administra las tecnologías de la información y la comunicación, siendo responsable de los aspectos técnicos, tecnológicos, científicos, de inteligencia de negocios y legales de bases de datos.

b) Programador. Profesional capacitado para desempeñarse en todos los campos de desarrollo de software. Asimila y profundiza en el conocimiento de diversos lenguajes de programación, sistemas operativos, y otras herramientas para el desarrollo de sistemas de información, manteniéndose actualizado en la dinámica de esta disciplina. Su labor es programar, implantar, gestionar, mantener y operar los sistemas de información computacionales requeridos por la empresa o institución, como también desempeñarse en el área de soporte de dichos sistemas.

c) Mercadeo. Se desempeñará eficaz y eficientemente en actividades que involucren las políticas, procesos y recursos de la mercadotecnia, y estará dispuesto a mantener una actividad participativa, reflexiva y creativa dentro del área de mercadeo. Está capacitado para desenvolverse en las funciones de investigación, planificación, organización, dirección y control de los recursos humanos y financieros. 


\section{Aspecto legal}

En El Salvador, al igual que en todos los países del mundo donde hay pymes, estas emplean un porcentaje mayoritario de la población económicamente activa, lo cual ha llevado a distintas entidades a escala internacional a promover el aumento de la competitividad individual para aumentar la empresarial y la de cada país en su conjunto.

a) Sistema de protección de datos.

b) Firma electrónica legal, certificados digitales.

c) Seguridad de la información.

\section{Sistemas transaccionales}

Los sistemas transaccionales son sistemas de información diseñados para recolectar, almacenar, modificar y recuperar todo tipo de información que sea generada por las transacciones en una organización (Ohlhorst, 2012). Dentro de la plataforma de sistemas transaccionales se encuentran los siguientes:
a) Sistemas de facturación
b) Sistemas de cuentas por cobrar
c) Sistemas de promoción

\section{Tecnología}

La eficiencia en el uso de las tecnologías de la información (TI) en las pymes son la clave del éxito; las posibilidades de su aplicación van desde gestión de documentos hasta oficinas virtuales. Las TI serán un punto crucial entre el crecimiento, la evolución y la trascendencia de una compañía, a cualquier nivel y en cualquier lugar.

En muchas ocasiones podemos apreciar que algunos negocios aún no entienden lo importante que es el uso de las tecnologías, lo que no les permite crecer como empresas y poder desarrollarse de una manera más eficiente en el mercado, con lo que están perdiendo la oportunidad de obtener mayores ganancias y obtener más clientes.

Las cuatros tecnologías más utilizadas en las pymes son las siguientes:

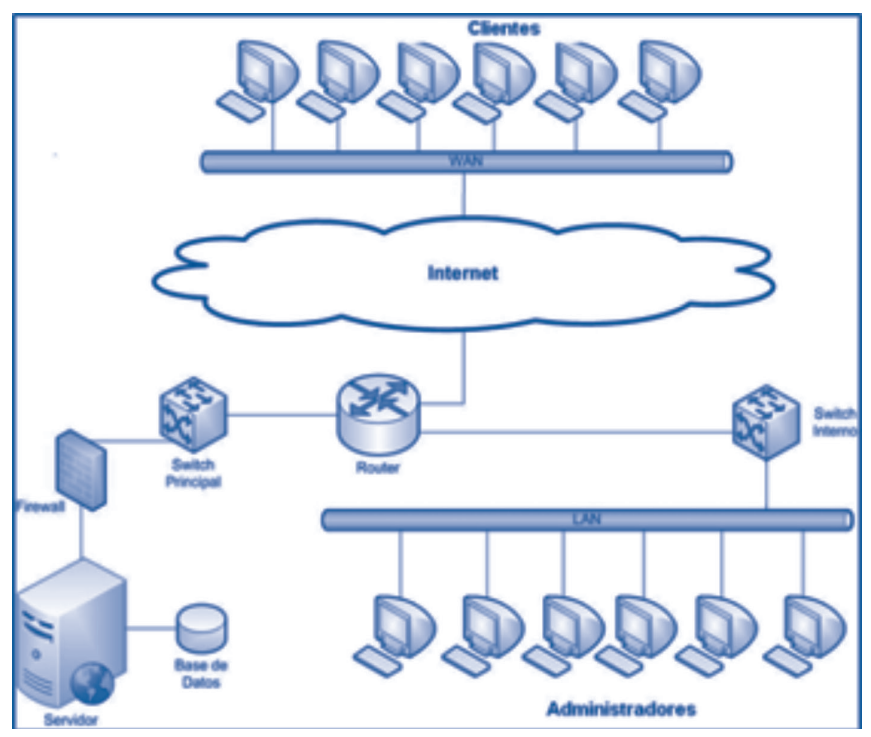

Figura 17.

Estructura de red.

Fuente: Sitio web www.centrallam.com.

a) Las aplicaciones de software empresarial

b) Comunicaciones

c) Búsqueda en internet

d) Negocios y hardware

e) Servidor estructura de red

\section{Medios sociales}

Cada vez más pymes están incluyendo los medios sociales dentro de su estrategia de mercadotecnia para promocionar sus productos, conocer las nuevas tendencias, conseguir nuevos clientes, interactuar con los clientes. Medios sociales es un término de mercadotecnia que hace referencia a la estrategia y conjunto de acciones llevadas a cabo en redes sociales y comunidades en línea con una finalidad publicitaria o comercial. Los tres principales medios sociales para ayudar con el crecimiento y apoyo de las pymes son Facebook, Twitter y Google.

\section{Mercadotecnia}

Esta tiene un papel fundamental para el éxito de las pymes y, por tanto, para el desarrollo de la economía. Nunca antes las empresas han tenido la oportunidad de tener a su disposición las mejores técnicas y herramientas de 
marketing y llegar a cualquier consumidor en cualquier lugar con un coste tan bajo, gracias a las nuevas tecnologías, a los medios sociales y a los nuevos dispositivos móviles que los posibilitan.

Mercadotecnia es un proceso social y administrativo porque interviene un conjunto de personas con sus inquietudes y necesidades; y porque necesita de una determinada cantidad de elementos, tales como la organización, la implantación y el control, para un desarrollo eficiente de las actividades. La única función que debe cumplir la mercadotecnia es alcanzar las metas que la empresa se plantea en lo que respecta a las ventas y distribución de sus productos o servicios, para que esta se mantenga vigente.

a) Sistemas de información

b) Sistema de planificación

c) Sistema de control

d) Sistema de lanzamiento de nuevos productos

\section{Estrategias}

Identifica las diferentes maneras de cómo los administradores pueden seleccionar las metas más adecuadas y alcanzarlas, por medio de un diseño del plan que permitirá lograr los objetivos particulares de la empresa.

Para poder desarrollar una estrategia acorde con las necesidades de una organización, es fundamental tomar en cuenta algunas variables y factores que determinan la funcionalidad y la eficiencia de dichas estrategias con base en la estructura de la compañía.

\section{La investigación propone dos planes}

a) Plan de implantación del modelo

b) Plan de validación del modelo

\section{Conclusiones}

Después de presentar los resultados, se concluye lo siguiente:

a) Se han identificado los actores que participan en los procesos de comercio electrónico. Con esto se facilitó la definición de los elementos que son parte del modelo de incorporación de la pymes al comercio electrónico.

b) Se determinó el escenario actual de las pymes, en el cual se puede hacer mención de que el universo de información que se maneja en las instituciones es inmenso; los datos no están totalmente organizados, lo que hace que las empresas no exploten óptimamente los segmentos de mercado que tienen y obtengan así altos niveles de productividad.

c) Con los insumos de la investigación se elaboró el modelo de incorporación de las pymes al comercio electrónico. Los elementos que conforman el modelo cubren todas las necesidades fundamentales para que las pymes puedan realizar de forma completa la migración de sus sistemas a un ambiente global de negocios y estén capacitadas para enfrentarse a los desafíos que demandan los nuevos mercados.

\section{Referencias}

Aguilar, L.J. (2013). Big Data, Analisis de los grandes volumenes de datos. Mexico: Alfaomega.

Castro, L. (16 de 3 de 2014). "Aprender Internet". Obtenido de http://aprenderinternet.about.com/od/ ConceptosBasico/a/Internet-de-las-cosas.htm

Gerencia, v. (13 de 03 de 2014). "Funciones gerenciales". Recuperado el 2 de 2014, de http://www.degerencia.com/ tema/comercio_electronico

IBM. (18 de 6 de 2012). "Tipos de datos de Big Data, Web y redes sociales". Recuperado el 2014, de http://www.ibm. com/developerworks/ssa/local/im/que-es-big-data/

Kaba, I. (2008). "Elementos básicos del comercio electrónico". Ciudad de la Habana: Universitaria. Obtenido de Elementos básicos del comercio electrónico: http:// www.ceaam.edu.mx/new/ni4/ce/MOD_2_LECT_1_ELEMENTOS_BASICOS_DE_COMERCIO_ELECTRONICO.pdf

Martínez, J.E. (2013). Desafio y oportunidades de las Pymes salvadoreñas. San Salvador: N/A.

Minec (2012). Estadisticas y Censos El Salvador. San Salvador: N/A.

"NIIF El Salvador" (26 de 02 de 2014). Obtenido de www. niifelsalvador.com/pymes.php

Ohlhorst, F. (2012). BIG DATA ANALYTICS. Turning Big Data into Big Money. Wiley \& Sas business series .

WCruzy (20 de 2 de 2014). "Seguridad de la informacion". Obtenido de http://wcruzy.uphero.com/iii/seguridadinformacion.pdf 